Check for updates

Cite this: RSC Adv., 2017, 7, 51055

Received 11th October 2017

Accepted 23rd October 2017

DOI: 10.1039/c7ra11220c

rsc.li/rsc-advances

\section{Enhancing intramolecular features and identifying defects in organic and hybrid nanoarchitectures on a metal surface at room temperature using a $\mathrm{NaCl}-$ functionalized scanning tunneling microscopy tip}

\begin{abstract}
David Peyrot and Fabien Silly iD *
Scanning tunneling microscopy (STM) is a powerful method to characterize two-dimensional organic structures and their local electronic properties. Intermolecular features are usually routinely revealed using STM at very low temperature. The resolution of STM images recorded at room temperature is in comparison quite limited. We investigate here the morphology and local electronic properties of organic and hybrid nanoarchitectures laying on metal surface at room temperature using STM with a classical Ptlr tip and a novel $\mathrm{NaCl}$-functionalised tip. STM images show that the $\mathrm{NaCl}$-functionalized tip allows revealing at room temperature intermolecular features and variation of molecular local electronic properties, that are not visible using a Ptlr tip. This new method opens new opportunities for characterizing and assessing at the atomic scale the morphology and electronic properties of organic nanoarchitectures on highly conductive surfaces not only at room temperature but also at low temperature.
\end{abstract}

\section{Introduction}

Characterizing two-dimensional nanostructures and nanoarchitectures $^{\mathbf{1 - 1 9}}$ at the atomic scale is a challenge of nanosciences for the development of new functionalized nanomaterials for nanotechnology. ${ }^{\mathbf{2 0 , 2 1}}$ Scanning probe microscopy (SPM) is a powerful technique to assess locally the topographic and electronic properties of nanostructured surfaces. During the last years intense research effort has been dedicated to improve the resolution of SPM techniques. The resolution of non-contact atomic force microscopy (nc-AFM) in vacuum at low temperature has been especially improved using functionalized SPM tips. Images of molecular carbon skeletons have been acquired at low temperature using Xe-functionalized and CO-functionalized tips. ${ }^{22-39}$ The apex of the tip is functionalized by flooding the vacuum chamber in gas when the tip temperature is below the gas condensation temperature. Gas molecules thus adsorb onto the tip apex. This technique has also been adapted to enhance the resolution of scanning tunneling microscopy (STM). Temirov et al. functionalized the STM tip apex with $\mathrm{H}_{2}$ and $\mathrm{D}_{2}$ molecules at low temperature. ${ }^{40}$ They showed that, using these tips, the STM contrast can be switched between the conventional mapping of the electronic local density of states and a new geometric imaging by selecting the appropriate tunneling bias voltage. PTCDA and tetracene molecular benzene rings appear as bright spots at low

CEA, IRAMIS, SPEC, TITANS, CNRS, Université Paris-Saclay, F-91191 Gif sur Yvette, France. E-mail: fabien.silly@cea.fr; Fax: +33 169088446; Tel: +33 169088019 temperature in the STM images using these functionalised tips. $^{\mathbf{4 0 - 4 2}}$ The main drawback of these AFM and STM techniques is that they require very low temperature to first functionalised the SPM tips and then to perform the characterization of the nanostructures. To achieve atomic scale electronic contrast in the SPM images of organic nanoarchitectures, molecules often have to lay on an insulating thin film, as $\mathrm{NaCl}$, to be electronically decoupled from the supporting metal surface. The challenge still consists to assess to the individual electronic properties of molecules at room temperature on metal surfaces. Intramolecular features have been observed in molecules adsorbed on metal surfaces using STM at room temperature but only using tunneling bias close to the Fermi level, ${ }^{\mathbf{4 3}}$ where the integral of the density of states is close to the local density of states. $^{44}$ Intermolecular features are usually lost when the tunneling bias is higher than few meV. The tunneling bias can thus not be used as a free parameter to optimize STM images, which is essential to discover new localized phenomena. Intramolecular features have been observed in molecules adsorbed on graphite, ${ }^{\mathbf{4 5 , 4 6}}$ where the electronic coupling at the interface is less strong than on a metal surface.

In this paper we investigate the contrast enhancement in the STM images of organic and hybrid nanoarchitectures on metal surfaces at room temperature induced by a NaCl-functionalized STM tip. Archetypal 2D model systems (PTCDI molecules ${ }^{47}$ and PTCDI-NaCl nanoarchitectures ${ }^{48}$ ) are selected as a benchmarks to assess the enhanced resolution of the $\mathrm{NaCl}$-functionalized STM tip in comparison with a non-functionalised PtIr STM tip. STM data reveal that new electronic features appear at room 
temperature in the STM images recorded with the $\mathrm{NaCl}$ functionalized STM tip.

\section{Experimental}

Experiments were performed in a ultrahigh vacuum (UHV) chamber at a pressure of $10^{-8}$ Pa. 3,4,9,10-Perylenetetracarboxylic diimide (PTCDI, $\mathrm{C}_{24} \mathrm{H}_{10} \mathrm{~N}_{2} \mathrm{O}_{4}$ ) molecules (Fig. 2a) and $\mathrm{NaCl}$ were evaporated at $250{ }^{\circ} \mathrm{C}$ and $390^{\circ} \mathrm{C}$ respectively onto a $\mathrm{Au}(111)$ surface. Cut PtIr tips were used to obtain constant current STM images at room temperature with a bias voltage applied to the sample. PtIr tips were cleaned and sharpen by applying pulses on the clean $\mathrm{Au}(111)$ surface. The PtIr tips were functionalized with $\mathrm{NaCl}$ by applying a bias pulse $(+3 \mathrm{~V})$ on top of an $\mathrm{NaCl}$ island. STM images were processed and analyzed using the FabViewer application. ${ }^{49}$

\section{Results}

\subsection{Enhanced STM contrast in $\mathrm{NaCl}$ island imaging}

The Fig. 1a presents an STM image of a crystalline $\mathrm{NaCl}$ island recorded first with a sharp PtIr tip (top) and then an $\mathrm{NaCl}$ functionalized STM tip (bottom) at room temperature. The yellow dashed line highlights the imaging transition between the two STM tips. The STM image reveals that the resolution of the STM image is drastically improved using the $\mathrm{NaCl}$ functionalized STM tip. The typical corrugation of the $\mathrm{NaCl}$ island taken along the line profiles indicated by the white arrow

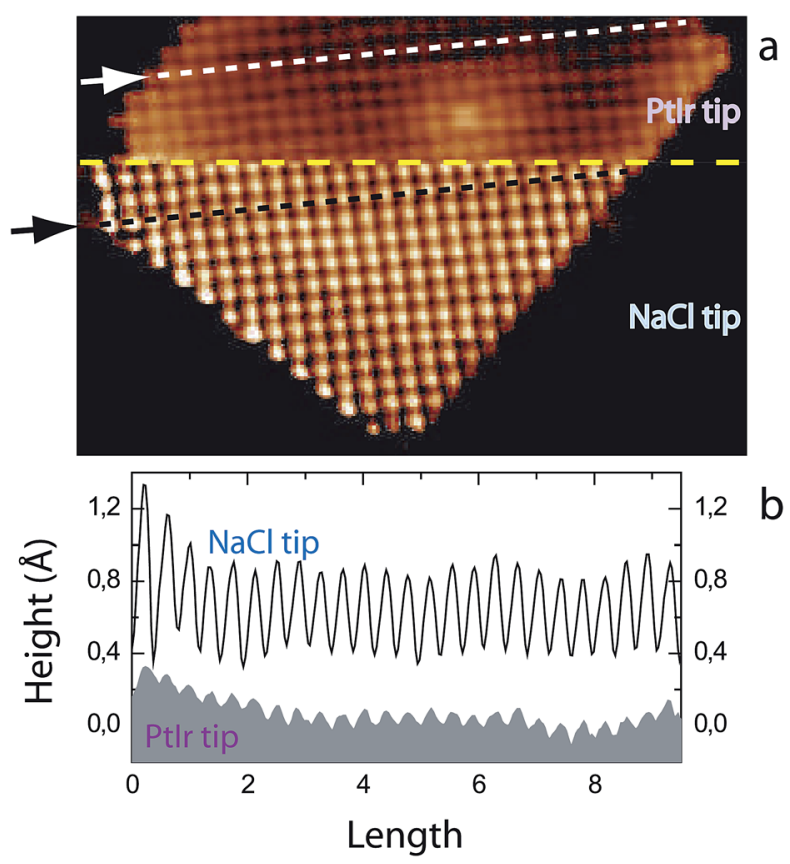

Fig. 1 (a) STM image of an crystalline $\mathrm{NaCl}$ island, $12 \times 7 \mathrm{~nm}^{2}, V_{\mathrm{s}}=$ $1.2 \mathrm{~V}, I_{\mathrm{t}}=106 \mathrm{pA}$. The yellow dash line highlights the transition between STM imaging using the PtIr STM tip (top) and the $\mathrm{NaCl}$ functionalized STM tip (bottom). (b) Line profiles taken along the gray dashed line (PtIr STM tip) and the black dashed line ( $\mathrm{NaCl}$ functionalized STM tip).
(PtIr STM tip) and the black arrow (NaCl-functionalized STM tip) is presented in Fig. 1b. The corrugation of the $\mathrm{NaCl}$ island is $\sim 0.07 \AA$ with the PtIr STM tip, whereas its is $\sim 0.50 \AA$ with the $\mathrm{NaCl}$ functionalized STM tip. The corrugation has been enhanced by a factor 7 using the NaCl-functionalized STM tip.

\subsection{Enhanced STM contrast in NaCl-PTCDI nanoarchitecture imaging}

The scheme of a 3,4,9,10-perylenetetracarboxylic diimide (PTCDI) molecule and the corresponding charge density contours of LUMO, LUMO + 1, HOMO, HOMO -1 are presented in Fig. 2. The charge density contours were calculated using complete neglect of differential overlap (CNDO) semi empirical method. This method uses two approximations: core approximation (only the outer valence electrons are explicitly included) and zero-differential overlap. ${ }^{50}$

3.2.1 Identifying molecules having different electronic properties. The Fig. 3 presents STM images of PTCDI molecules (a and b) and PTCDI mixed with $\mathrm{NaCl}(\mathrm{c}-\mathrm{f}$ ) at room temperature. The STM images in Fig. 3a, c, e and $g$ have been recorded using a sharp PtIr tip, whereas the STM images in Fig. $3 \mathrm{~b}, \mathrm{~d}, \mathrm{f}$ and $\mathrm{h}$ have been recorded using an NaCl-functionalized tip. The STM images reveal that most of the PTCDI molecules adopt a capsule-shape with the PtIr tip, whereas they adopt a pronounced peanut-contour with the NaCl-functionalized tip,
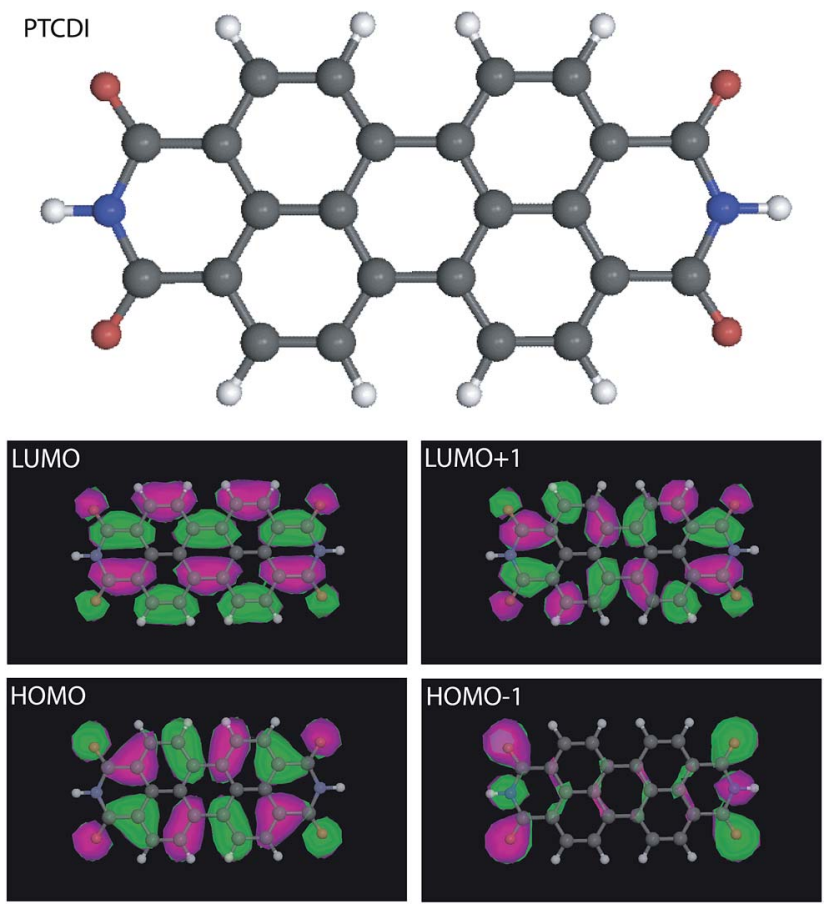

Fig. 2 Scheme of 3,4,9,10-perylenetetracarboxylic diimide (PTCDI) molecule (top) and calculated densities of unoccupied states (bottom). (Bottom) Image of the molecular orbitals (charge density contours) corresponding to the LUMO, LUMO + 1, HOMO, HOMO - 1 superimposed to the PTCDI skeleton (gray balls are carbon atoms, red balls are oxygen atoms, white balls are hydrogen atoms, and blue balls are nitrogen atoms). The different colors in the HOMO and LUMO maps (bottom) mean different signs. 


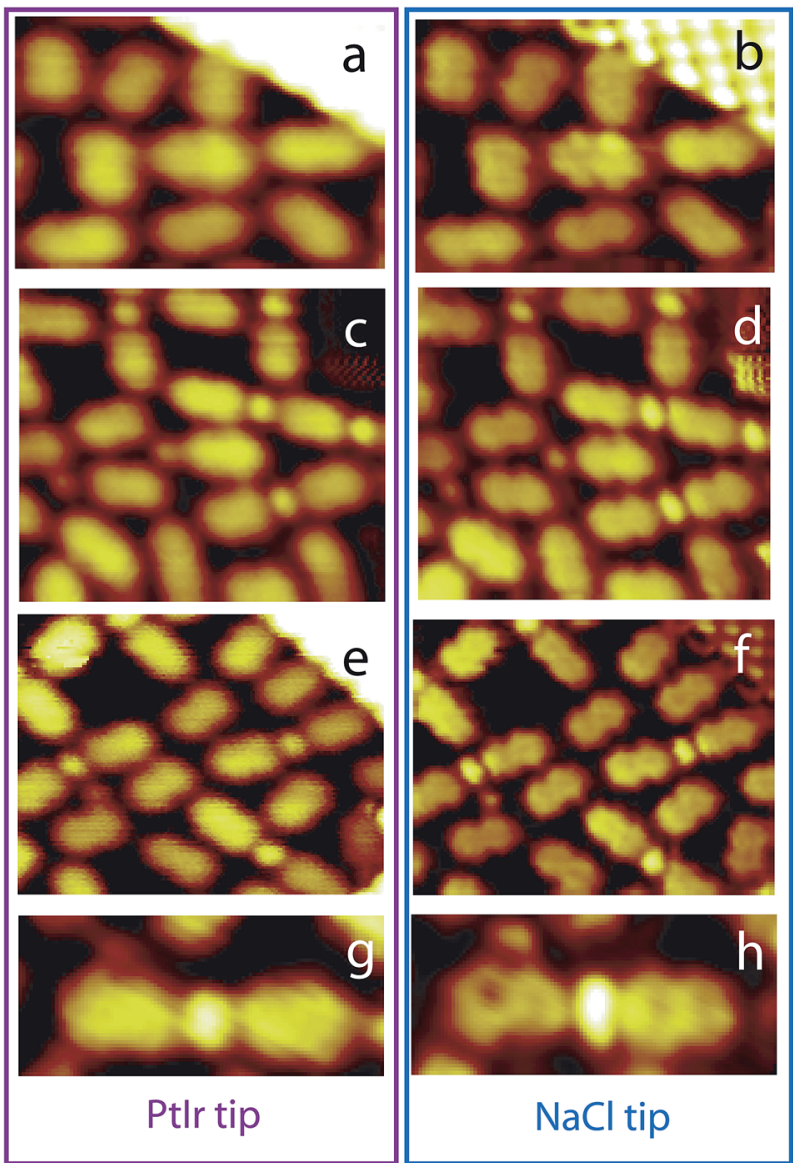

Fig. 3 STM image of PTCDI molecules at room temperature, using a PtIr tip (a) and an $\mathrm{NaCl}$ functionalized tip (b), $5 \times 3 \mathrm{~nm}^{2}, V_{\mathrm{s}}=1.2 \mathrm{~V}, I_{\mathrm{t}}=$ 106 pA. STM images of PTCDI mixed with $\mathrm{NaCl}$ at room temperature, using a PtIr tip ( $c$ and e) and an $\mathrm{NaCl}$ functionalized tip ( $d$ and f), $5 \times 5$ $\mathrm{nm}^{2}, V_{\mathrm{s}}=1.2 \mathrm{~V}, l_{\mathrm{t}}=106 \mathrm{pA}$. STM images of a PTCDI $\cdots \mathrm{NaCl} \cdots \mathrm{PTCDI}$ stick at room temperature, using a PtIr tip (g) and an $\mathrm{NaCl}$ functionalized tip (h), $5 \times 2 \mathrm{~nm}^{2}, l_{\mathrm{t}}=106 \mathrm{pA}$.

Fig. $3 \mathrm{~b}, \mathrm{~d}$, $\mathrm{f}$ and $\mathrm{h}$. Additional details are also revealed inside the molecules using the NaCl-functionalized tip. Two dark round areas are observed inside the PTCDI molecules. These dark areas are not observed using the PtIr tip, Fig. 3a, c, e, g. The PTCDI molecules are thus adopting a " 8 "-shape when the $\mathrm{NaCl}$ functionalized tip is used. This " 8 "-shape can be observed in Fig. $3 \mathrm{~b}, \mathrm{~d}$ and $\mathrm{f}$ but it is especially visible in the Fig. $3 \mathrm{~h}$, where the color scale bar has been specifically adjusted. This " 8 "shape is similar to the molecular LUMO +1 shape (Fig. 2). In contrast with these molecules, the central molecule in Fig. 3a adopts a non-uniform shape in the STM image recorded using the PtIr tip. The STM image obtained using the NaClfunctionalized tip reveals that this molecule adopts a shape that is similar to the molecular LUMO shape (Fig. 2). The NaClfunctionalized tip thus reveals that PTCDI molecules on $\mathrm{Au}(111)$ can adopt the LUMO or the LUMO + 1 shape in the STM image. In comparison the $\mathrm{NaCl}$ dimers appear as bright spots in the STM images using the PtIr and the NaCl-functionalized tips.

3.2.2 Bias-dependent STM contrast. Fig. 4 shows STM images of the NaCl-PTCDI "mesh"-nanoarchitecture on

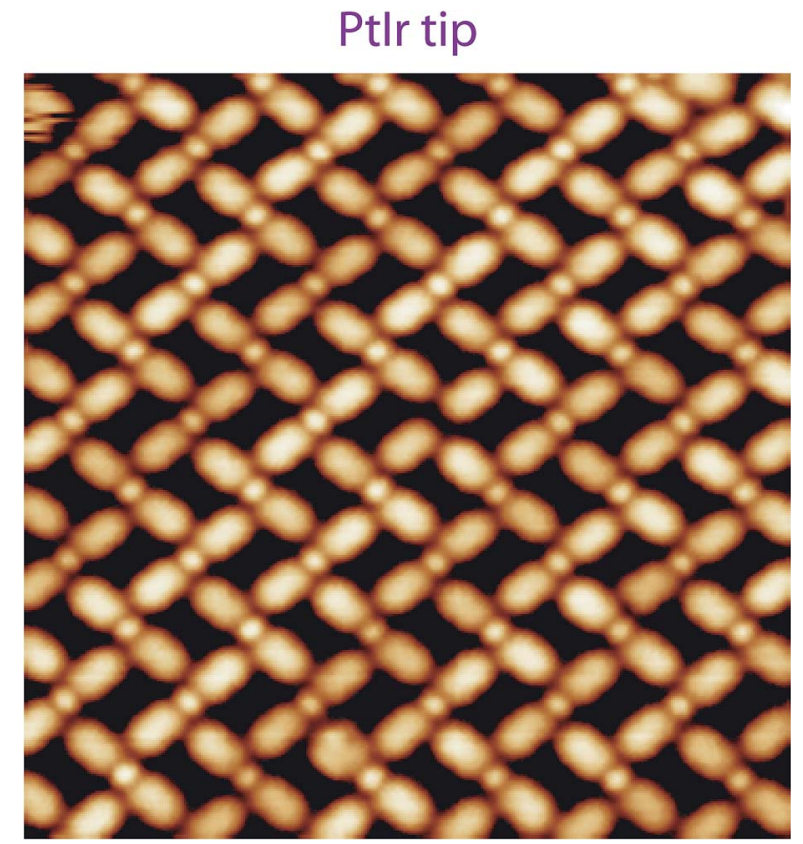

Fig. 4 STM image of the PTCDI-NaCl mesh-nanoarchitecture ${ }^{48}$ recorded at room temperature using a clean PtIr STM tip, $14 \times 14 \mathrm{~nm}^{2}$, $V_{\mathrm{s}}=+1.3 \mathrm{~V}, I_{\mathrm{t}}=180 \mathrm{pA}$.

$\mathrm{Au}(111)^{48}$ recorded using a clean and sharp PtIr tip. This structure is formed after depositing PTCDI and $\mathrm{NaCl}$ on $\mathrm{Au}(111)$ and the surface post-annealed at $100{ }^{\circ} \mathrm{C}$. The PTDI molecules appear as capsules and $\mathrm{NaCl}$ dimers as bright spots, independently of the selected tunneling bias. We did not find any tunneling conditions that allow imaging intermolecular details using a clean PtIr tip, as it can be seen in the additional STM images presented in ref. 48 .

Fig. 5 shows STM images of NaCl-PTCDI "mesh"-nanoarchitecture on $\mathrm{Au}(111)$ recorded at room temperature using an $\mathrm{NaCl}$ functionalized $\mathrm{NaCl}$ tip. The STM images are sequentially

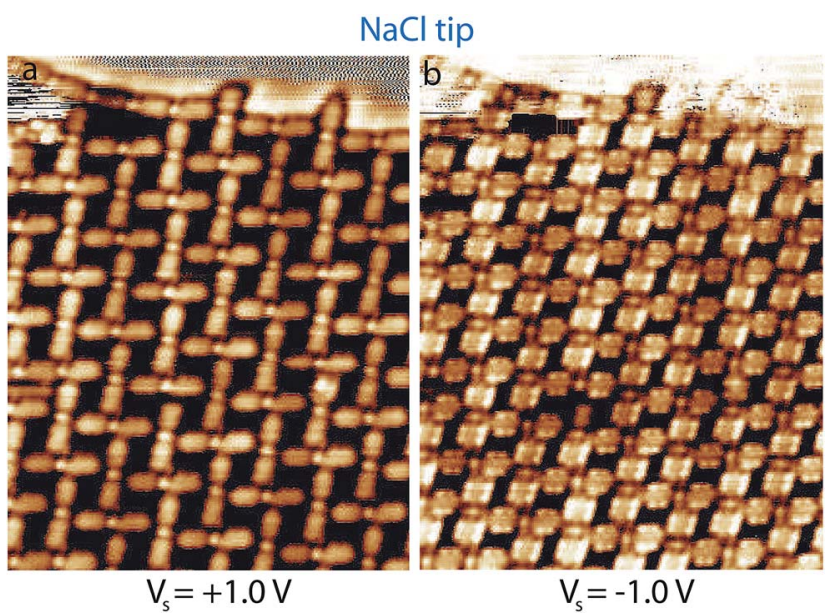

Fig. 5 Large scale STM image of the PTCDI-NaCl mesh-nanoarchitecture at room temperature using an $\mathrm{NaCl}$ functionalized STM tip, $18 \times 14 \mathrm{~nm}^{2}$, (a) $V_{\mathrm{s}}=+1.0 \mathrm{~V}, I_{\mathrm{t}}=365 \mathrm{pA}$; (b) $V_{\mathrm{s}}=-1.0 \mathrm{~V}, \mathrm{I}_{\mathrm{t}}=365 \mathrm{pA}$. 
recorded at two different tunneling bias ((a) $V_{\mathrm{s}}=+1.0 \mathrm{~V}$ and (b) $\left.V_{\mathrm{s}}=-1.0 \mathrm{~V}\right)$. The STM images show that the appearance of the hybrid nanoarchitecture depends drastically of the tunneling bias.

High resolution STM images of the NaCl-PTCDI "mesh"nanoarchitecture sequentially recorded using an $\mathrm{NaCl}-$ functionalized tip at $V_{\mathrm{s}}=+1.0 \mathrm{~V}$ (a) and $V_{\mathrm{s}}=-1.0 \mathrm{~V}$ (b) are presented in Fig. 6 . The network unit cell shape is not varying
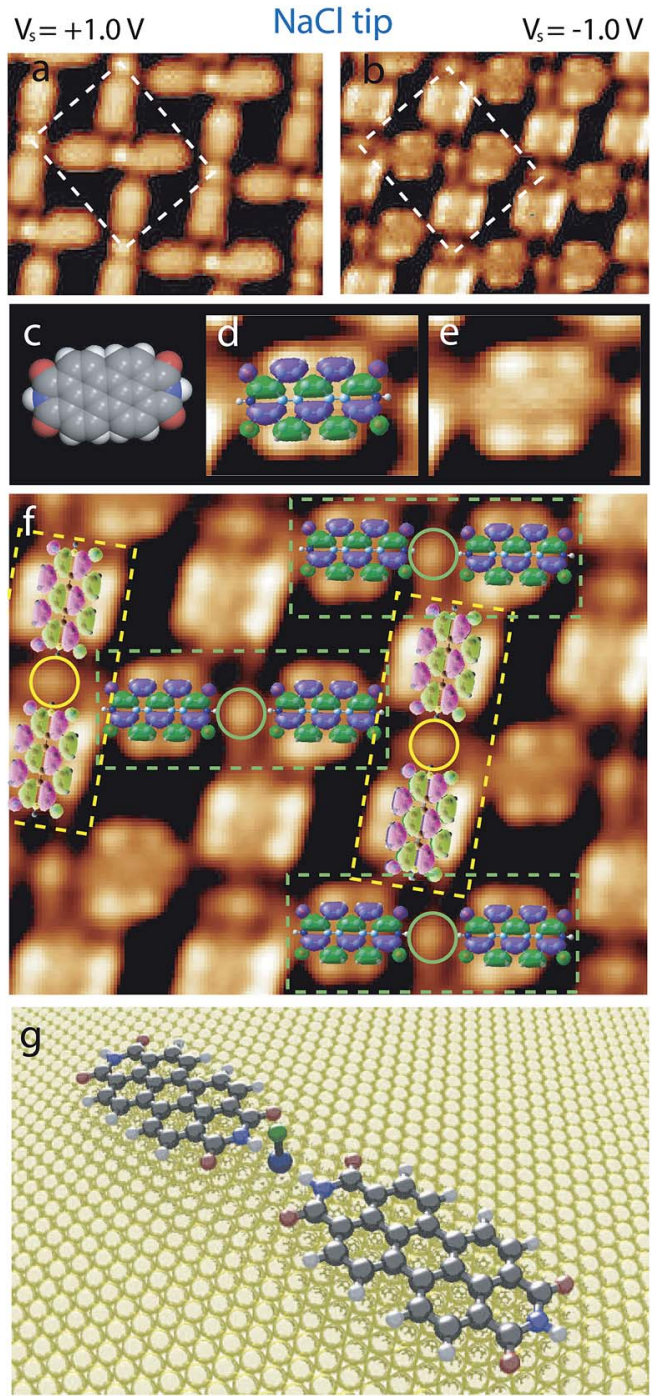

Fig. 6 STM image of the PTCDI-NaCl mesh-nanoarchitecture at room temperature and a post annealing at $100{ }^{\circ} \mathrm{C}$ using an $\mathrm{NaCl}$ functionalized STM tip, $5 \times 4 \mathrm{~nm}^{2}$, (a) $V_{\mathrm{s}}=+1.0 \mathrm{~V}, I_{\mathrm{t}}=365 \mathrm{pA}$; (b) $V_{\mathrm{s}}=$ $-1.0 \mathrm{~V}, I_{\mathrm{t}}=365 \mathrm{pA}$. (c) Scheme of the PTCDI molecule. (d) Charge density contours (blue and green color) of the PTCDI LUMO superimposed to the STM image of the molecule (e). (e) High resolution STM image of the PTCDI molecule, $2 \times 1.5 \mathrm{~nm}^{2}, V_{\mathrm{s}}=-1.0 \mathrm{~V}, I_{\mathrm{t}}=365 \mathrm{pA}$. (f) Model of the $\mathrm{NaCl}-\mathrm{PTCDI}$ mesh-arrangement. Charge density contours (blue and green color, yellow and pink) of the PTCDI LUMO have been superimposed to the STM image (b). Parallel PTCDI... $\mathrm{NaCl}$...PTCDI sticks are highlighted by dashed green and yellow rectangles respectively. $\mathrm{NaCl}$ is highlighted by green and yellow circles. (g) Model of the PTCDI... NaCl...PTCDI stick on $\mathrm{Au}(111) . \mathrm{NaCl}$ dimer is represented with a dimer composed of a blue (chlorine) and a green (sodium) ball. with the tunneling bias. The network unit cell of this porous structure is a rectangle with $2.3 \mathrm{~nm}$ and $2.5 \mathrm{~nm}$ unit cell constants and an angle of $\sim 90^{\circ}$ between the axes (Fig. 6a and b). The STM images show that PTCDI molecules adopt a " 8 "-shape $(\mathrm{LUMO}+1)$ for $V_{\mathrm{s}}=+1.0 \mathrm{~V}$, whereas the molecules adopt the molecular LUMO shape for $V_{\mathrm{s}}=-1.0 \mathrm{~V}$ (see comparison between molecular skeleton, molecular LUMO and the STM image of one molecule in Fig. 6c-e). The orientation of the PTCDI molecules can be determined with a high accuracy from the high resolution STM image recorded at $V_{\mathrm{S}}=-1.0 \mathrm{~V}$ using the NaCl-functionalized tip, Fig. $6 \mathrm{~b}$ and f. One $\mathrm{N}-\mathrm{H}$ group of the PTCDI molecules is pointing toward a $\mathrm{NaCl}$ dimer forming straight PTCDI $\cdots \mathrm{NaCl} \cdots$ PTCDI sticks (highlighted by dashed green and yellow rectangles in Fig. 6f). A model of PTCDI $\cdots$ $\mathrm{NaCl} \cdots$ PTCDI stick is presented in Fig. $6 \mathrm{~g}$. The second PTCDI $\mathrm{N}-\mathrm{H}$ group is in comparison pointing toward an oxygen atom of a neighboring molecule, forming an $\mathrm{N}-\mathrm{H} \cdots \mathrm{O}$ hydrogen-bond, Fig. 6f. The angle between neighboring PTCDI $\cdots \mathrm{NaCl} \cdots \mathrm{PTCDI}$ sticks is $\sim 80^{\circ}$.

3.2.3 Identifying variation of local electronic properties in 2D hybrid nanoarchitecture. High resolution STM images of a local defect in the NaCl-PTCDI "mesh"-nanoarchitecture recorded using an NaCl-functionalized tip at $V_{\mathrm{S}}=+1.0 \mathrm{~V}$ (a) and $V_{\mathrm{s}}=-1.0 \mathrm{~V}(\mathrm{~b})$ are presented in Fig. 7. All the PTCDI molecules look nearly identical for $V_{\mathrm{s}}=+1.0 \mathrm{~V}$ (Fig. 7a). In contrast the STM image recorded at $V_{\mathrm{s}}=-1.0 \mathrm{~V}$ reveals that one molecule (highlighted by a dashed yellow rectangle in Fig. 7) differs from the other one. No intramolecular feature can be observed in this molecule at $V_{\mathrm{s}}=-1.0 \mathrm{~V}$, whereas all the other molecules adopt

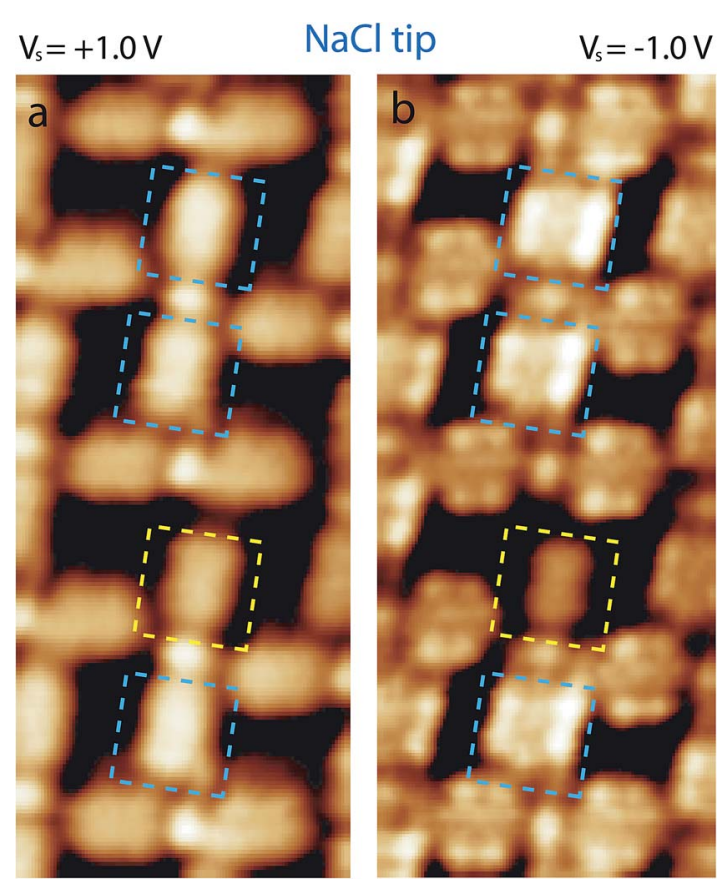

Fig. 7 STM image of local defect in the PTCDI-NaCl mesh-nanoarchitecture at room temperature using an $\mathrm{NaCl}$ functionalized STM tip, $7 \times 4 \mathrm{~nm}^{2}$, (a) $V_{\mathrm{s}}=+1.0 \mathrm{~V}, I_{\mathrm{t}}=365 \mathrm{pA}$; (b) $V_{\mathrm{s}}=-1.0 \mathrm{~V}, I_{\mathrm{t}}=365 \mathrm{pA}$. Molecular defect is highlighted by a dash yellow rectangle. Regular molecules are highlighted by a dash blue rectangles for comparison. 
a LUMO-shape. This specific molecule appears to be located further to the neighboring PTCDI molecule, resulting in a different intermolecular interactions. Subtle variations in nanoobject lateral binding can drastically affect its electronic properties. ${ }^{51}$ We recently showed that variation of lateral coupling in PTCDI assemblies can lead to a drastic modification of molecular appearance in the STM images. ${ }^{52}$ The NaCl functionalised tip therefore reveals variation of molecular electronic properties either induced by intermolecular coupling or due to a tiny defect in the molecular structure.

\section{Discussion}

These experimental observations show that $\mathrm{NaCl}$ functionalized STM tip can be used to probe the local electronic properties of two-dimensional nanostructures on metal surface at room temperature. The STM images reveal that an increase of contrast $(700 \%)$ has been achieved when imaging an $\mathrm{NaCl}$ island, Fig. 1. The NaCl-functionalized tip allows revealing at room temperature intramolecular features in organic nanoarchitectures deposited on $\mathrm{Au}(111)$. For example PTCDI molecules adopt a capsule-shape in the STM images recorded using a conventional PtIr tip. In contrast the molecules usually adopt a 8-shape in the STM images recorded using a NaClfunctionalized tip, Fig. 3. This 8 -shape is similar to the molecular LUMO + 1 (Fig. 2). Individual molecules have a specific electronic behavior can be undoubtedly identified in the STM images recorded using the NaCl-functionalized tip. For example the PTCDI molecule in the center of the Fig. 3a has an inhomogeneous shape in the STM image recorded using the PtIr STM tip. The STM image recorded using the NaClfunctionalized tip reveals that the shape of this molecule is similar to the LUMO of the molecule, whereas the shape of the other molecules is similar to the LUMO +1 , Fig. $3 \mathrm{~b}$. This highlights that molecular assembly is drastically affecting the electronic properties of the molecular arrangement on metal surfaces, and this effect can be observed at room temperature using STM. High-resolution STM tips are however required to reveal this phenomenon.

Additional contrast can be obtained with the NaCl STM tip by changing the tunneling bias, Fig. 6 . The high resolution STM images in Fig. 6 show that the PTCDI molecules adopt the LUMO +1 shape at $V_{\mathrm{s}}=+1.0 \mathrm{~V}$, whereas the molecules adopt the LUMO shape at $V_{\mathrm{s}}=-1.0 \mathrm{~V}$. The sophisticated contrast of the molecular LUMO allows precisely determining the orientation of all the PTCDI molecules in the hybrid PTCDI-NaCl nanoarchitecture. Surprisingly the molecular LUMO is observed in the STM images far from the Fermi level $\left(V_{\mathrm{s}}=-1.0 \mathrm{~V}\right)$. Cao et al. however showed that the LUMO of perylene derivatives (very similar to PTCDI molecules) is localized below the $\mathrm{Au}(111)$ surface Fermi level. This broad state has nevertheless a contribution close to the Fermi level. ${ }^{53}$ This explains why intramolecular details in STM images are usually observed close to the Fermi level, where the integral of the density of states is close to the local density of states. ${ }^{44}$ The appearance of the LUMO below $E_{\mathrm{F}}$ is usually attributed to a more effective core hole self-screening by the excited electron in the $\pi^{*}$ molecular orbital compared to the core hole screening by metal electrons. ${ }^{53}$ Far from the Fermi level, other surface electronic contributions, such as surface states of (111) noble metal surfaces ${ }^{44}$ are weakening the contribution of the molecules in the STM signal. It is therefore very surprising to observe intermolecular features in the STM images far from the Fermi level, especially at room temperature. Recording intermolecular features usually requires STS mapping at low temperature with the classic PtIr or W STM tip. This is one of the most interesting benefit of the $\mathrm{NaCl}$ functionalised STM tip. Defects in the molecular arrangement can be undoubtedly identified in these high resolution images, i.e. the shape of single molecules can differ. The STM image in Fig. 7 shows that the NaClfunctionalized tip permits to precisely identify molecules having an unusual electronic behavior.

As there is no experimental image of the tip, it is experimentally impossible to assure which $\mathrm{NaCl}$ species is driving the enhanced resolution of the $\mathrm{NaCl}$-functionalized tip. STM images are however showing that $\mathrm{Cl}$ anions are appearing as a bright spots in the STM images, whereas Na cations are invisible (Fig. 1 and 5). This indicates that $\mathrm{Cl}$ cation density of states is having a high contrast in STM imaging. This therefore suggests that this species is most probably at the origin of the enhanced resolution of the NaCl-functionalised tip. The NaClfunctionalised STM tip was achieved numerous times using different PtIr tips, over months. Functionalizing the PtIr tip with $\mathrm{NaCl}$ did not appear to be more difficult than sharpening a PtIr tip with bias pulse. The $\mathrm{NaCl}$ functionalized-tip is able to reveal intramolecular details in the carbon skeleton of PTCDI molecules at room temperature, so this imaging method should also provide enhanced STM images of numerous carbon-based molecules, as CO-functionalized AFM tip is providing enhanced AFM images of numerous carbon-based molecules at very low temperature. The main drawn-back of this technique is that it requires depositing $\mathrm{NaCl}$ on the surface. $\mathrm{NaCl}$ self-assembles into flat crystalline islands on metal surfaces. $\mathrm{NaCl}$ islands have been indeed successfully grown on numerous crystalline metal surfaces, such as $\mathrm{Cu}(111),{ }^{54-56} \mathrm{Cu}(110),{ }^{54,57} \mathrm{Cu}(311),{ }^{57}$ $\mathrm{Ag}(111),{ }^{58} \mathrm{Ag}(100),{ }^{59} \mathrm{Ge}(100),{ }^{60} \mathrm{Al}(111),{ }^{61} \mathrm{Au}(111){ }^{62}$ and $\mathrm{Al}(100),{ }^{63}$ and so it is not polluting the metal surface. $\mathrm{NaCl}$ is however reacting with water molecules so high vacuum is required. Our experimental observations should motivate the development of detailed theoretical analysis of the use of NaCl-functionalized conductive tips in scanning tunneling microscopy as it was recently done with carbon-based tips?

\section{Conclusion}

To summarize, we investigated the properties of organic nanostructures on metal surface at room temperature using STM with a conventional PtIr tip and a NaCl-functionalized tip. We observed that the contrast in the STM images is greatly increased using the NaCl-functionalized tip. The NaClfunctionalized tip allows imaging at room temperature the electronic local density of states of the molecules and their precise orientation by selecting the appropriate bias tunneling voltage. The NaCl-functionalised STM tip also permits to 
identify local topographic and electronic defects in 2D nanoarchitectures at room temperatures. This new method opens new opportunities for characterizing and assessing at the atomic scale and at room temperature the morphology and electronic properties of organic nanoarchitectures on highly conductive surfaces. The NaCl-functionalized tip should not be limited to the STM technique but should also provide enhanced resolution in non-conductive atomic force microscopy (nc-AFM) technique. This new technique should stimulate theoretical developments to model the local electronic details observed in the STM images recorded using a NaCl-functionalized tip.

\section{Conflicts of interest}

There are no conflicts to declare.

\section{Acknowledgements}

The research leading to these results has received funding from the European Research Council under the European Union's Seventh Framework Programme (FP7/2007-2013)/ERC grant agreement no. 259297.

\section{References}

1 J. V. Barth, Annu. Rev. Phys. Chem., 2007, 58, 375-407.

2 X. Sun, F. Lafolet, G. Lemercier, F. Maurel and J.-C. Lacroix, J. Phys. Chem. C, 2017, 121, 20925-20930.

3 V. Q. Nguyen, X. Sun, F. Lafolet, J.-F. Audibert, F. Miomandre, G. Lemercier, F. Loiseau and J.-C. Lacroix, J. Am. Chem. Soc., 2016, 138, 9381-9384.

4 V. A. Gorbunov, S. S. Akimenko and A. V. Myshlyavtsev, Phys. Chem. Chem. Phys., 2017, 19, 17111-17120.

5 G. Pawin, K. L. Wong, K.-Y. Kwon and L. Bartels, Science, 2006, 313, 961-962.

6 S. Uemura, M. Aono, T. Komatsu and M. Kunitake, Langmuir, 2011, 27, 1336-1340.

7 T. Chen, W.-H. Yang, D. Wang and L.-J. Wan, Nat. Commun., 2013, 4, 1389.

8 H. Liang, W. Sun, X. Jin, H. Li, J. Li, X. Hu, B. K. Teo and K. Wu, Angew. Chem., Int. Ed., 2011, 50, 7562-7566.

9 J. A. Gardener, O. Y. Shvarova, G. A. D. Briggs and M. R. Castell, J. Phys. Chem. C, 2010, 114, 5859-5866.

10 L. Xu, X. Miao, B. Zha and W. Deng, Chem.-Asian J., 2013, 8, 926-933.

11 C. Tanioku, K. Matsukawa and A. Matsumoto, ACS Appl. Mater. Interfaces, 2013, 5, 940-948.

12 Y. Hu, K. Miao, B. Zha, L. Xu, X. Miao and W. Deng, Phys. Chem. Chem. Phys., 2015, 18, 624-634.

13 Y. Hu, K. Miao, S. Peng, B. Zha, L. Xu, X. Miao and W. Deng, CrystEngComm, 2016, 18, 3019-3032.

14 Q. Shen, J. H. He, J. L. Zhang, K. Wu, G. Q. Xu, A. T. S. Wee and W. Chen, J. Chem. Phys., 2015, 142, 101902.

15 E. Sierda, M. Abadia, J. Brede, M. Elsebach, B. Bugenhagen, M. H. Prosenc, M. Bazarnik and R. Wiesendanger, ACS Nano, 2017, 11, 9200-9206.
16 H. Y. Gao, Q. J. Shen, X. R. Zhao, X. Q. Yan, X. Pang and W. J. Jin, J. Mater. Chem., 2012, 22, 5336-5343.

17 M. A. Mezour, R. M. Choueiri, O. Lukoyanova, R. B. Lennox and D. F. Perepichka, Nanoscale, 2016, 8, 16955-16962.

18 J. Shang, Y. Wang, M. Chen, J. Dai, X. Zhou, J. Kuttner, G. Hilt, X. Shao, J. M. Gottfried and K. Wu, Nat. Chem., 2015, 7, 389-393.

19 D. Écija, R. Otero, L. Sánchez, J. M. Gallego, Y. Wang, M. Alcamí, F. Martín, N. Martín and R. Miranda, Angew. Chem., Int. Ed., 2007, 46, 7874-7877.

20 S. Yagai, M. Yamauchi, A. Kobayashi, T. Karatsu, A. Kitamura, T. Ohba and Y. Kikkawa, J. Am. Chem. Soc., 2012, 134, 18205-18208.

21 S. Yagai, Bull. Chem. Soc. Jpn., 2015, 88, 28-58.

22 S. Wickenburg, J. Lu, J. Lischner, H.-Z. Tsai, A. A. Omrani, A. Riss, C. Karrasch, A. Bradley, H. S. Jung, R. Khajeh, D. Wong, K. Watanabe, T. Taniguchi, A. Zettl, A. H. C. Neto, S. G. Louie and M. F. Crommie, Nat. Commun., 2016, 7, 13553.

23 L. Gross, F. Mohn, N. Moll, P. Liljeroth and G. Meyer, Science, 2009, 325, 1110-1114.

24 H.-Z. Tsai, A. A. Omrani, S. Coh, H. Oh, S. Wickenburg, Y.-W. Son, D. Wong, A. Riss, H. S. Jung, G. D. Nguyen, G. F. Rodgers, A. S. Aikawa, T. Taniguchi, K. Watanabe, A. Zettl, S. G. Louie, J. Lu, M. L. Cohen and M. F. Crommie, ACS Nano, 2015, 9, 12168-12173.

25 N. Moll, L. Gross, F. Mohn, A. Curioni and G. Meyer, New J. Phys., 2010, 12, 125020.

26 L. Gross, F. Mohn, N. Moll, G. Meyer, R. Ebel, W. M. AbdelMageed and M. Jaspars, Nat. Chem., 2010, 2, 821-825.

27 N. Pavlicek, B. Fleury, M. Neu, J. Niedenführ, C. HerranzLancho, M. Ruben and J. Repp, Phys. Rev. Lett., 2012, 108, 086101.

28 L. Gross, F. Mohn, N. Moll, B. Schuler, A. Criado, E. Guitián, D. Pena, A. Gourdon and G. Meyer, Science, 2012, 337, 13261329.

29 D. Hayes, G. B. Griffin and G. S. Engel, Science, 2013, 340, 1431-1434.

30 S. Kawai, A. S. Foster, T. Björkman, S. Nowakowska, J. Björk, F. F. Canova, L. H. Gade, T. A. Jung and E. Meyer, Nat. Commun., 2016, 7, 11559.

31 S. P. Jarvis, Int. J. Mol. Sci., 2015, 16, 19936-19959.

32 B. Schuler, G. Meyer, D. Peña, O. C. Mullins and L. Gross, J. Am. Chem. Soc., 2015, 137, 9870-9876.

33 K. Iwata, S. Yamazaki, P. Mutombo, P. Hapala, M. Ondriek, P. Jelinek and Y. Sugimoto, Nat. Commun., 2015, 6, 7766.

34 F. Albrecht, N. Pavliček, C. Herranz-Lancho, M. Ruben and J. Repp, J. Am. Chem. Soc., 2015, 137, 7424-7428.

35 S. Kawai, A. Sadeghi, F. Xu, L. Peng, A. Orita, J. Otera, S. Goedecker and E. Meyer, ACS Nano, 2015, 9, 2574-2583.

36 M. Corso, M. Ondrácek, C. Lotze, P. Hapala, K. J. Franke, P. Jelínek and J. I. Pascual, Phys. Rev. Lett., 2015, 115, 136101.

37 P. Hapala, M. Svec, O. Stetsovych, N. J. van der Heijden, M. Ondracek, J. van der Lit, P. Mutombo, I. Swart and P. Jelinek, Nat. Commun., 2016, 7, 11560.

38 M. Kim and J. R. Chelikowsky, Appl. Phys. Lett., 2015, 107, 163109. 
39 Z. Majzik, A. B. Cuenca, N. Pavlićek, N. Miralles, G. Meyer, L. Gross and E. Fernandez, ACS Nano, 2016, 10, 5340-5345.

40 R. Temirov, S. Soubatch, O. Neucheva, A. C. Lassise and F. S. Tautz, New J. Phys., 2008, 10, 053012.

41 C. Weiss, C. Wagner, C. Kleimann, M. Rohlfing, F. S. Tautz and R. Temirov, Phys. Rev. Lett., 2010, 105, 086103.

42 J. I. Martínez, E. Abad, C. González, F. Flores and J. Ortega, Phys. Rev. Lett., 2012, 108, 246102.

43 L. E. Dinca, F. De Marchi, J. M. MacLeod, J. Lipton-Duffin, R. Gatti, D. Ma, D. F. Perepichka and F. Rosei, Nanoscale, 2015, 7, 3263-3269.

44 M. Pivetta, F. Silly, F. Patthey, J. P. Pelz and W.-D. Schneider, Phys. Rev. B, 2003, 67, 193402.

45 F. Silly, J. Phys. Chem. C, 2014, 118, 11975-11979.

46 W. Chen, D.-C. Qi, H. Huang, X. Gao and A. T. S. Wee, Adv. Funct. Mater., 2011, 21, 410-424.

47 M. Mura, F. Silly, G. A. D. Briggs, M. R. Castell and L. N. Kantorovich, J. Phys. Chem. C, 2009, 113, 21840-21848.

48 J. Hieulle, D. Peyrot, Z. Jiang and F. Silly, Chem. Commun., 2015, 51, 13162-13165.

49 F. Silly, J. Microsc., 2009, 236, 211-218.

50 J. A. Pople and D. L. Beveridge, Approximate Molecular Orbital Theory, McGraw-Hill, 1970.

51 A. O. Gusev, A. Taleb, F. Silly, F. Charra and M.-P. Pileni, Adv. Mater., 2000, 12, 1583-1587.

52 J. Hieulle and F. Silly, J. Mater. Chem. C, 2013, 1, 4536-4539.
53 L. Cao, Y.-Z. Wang, T.-X. Chen, W.-H. Zhang, X.-J. Yu, K. Ibrahim, J.-O. Wang, H.-J. Qian, F.-Q. Xu, D.-C. Qi and A. T. S. Wee, J. Chem. Phys., 2011, 135, 174701.

54 J. Repp, G. Meyer, F. E. Olsson and M. Persson, Science, 2004, 305, 493.

55 F. E. Olsson, S. Paavilainen, M. Persson, J. Repp and G. Meyer, Phys. Rev. Lett., 2007, 98, 176803.

56 R. Bennewitz, A. S. Foster, L. N. Kantorovich, M. Bammerlin, C. Loppacher, S. Schär, M. Guggisberg, E. Meyer and A. L. Shluger, Phys. Rev. B: Condens. Matter Mater. Phys., 2000, 62, 2074-2084.

57 F. E. Olsson, M. Persson, A. G. Borisov, J.-P. Gauyacq, J. Lagoute and S. Fölsch, Phys. Rev. Lett., 2004, 93, 206803.

58 L. Ramoino, M. von Arx, S. Schintke, A. Baratoff, H. J. Güntherodt and T. A. Jung, Chem. Phys. Lett., 2006, 417, 22-27.

59 M. Pivetta, F. Patthey, M. Stengel, A. Baldereschi and W.-D. Schneider, Phys. Rev. B, 2005, 72, 115404.

60 K. Glöckler, M. Sokolowski, A. Soukopp and E. Umbach, Phys. Rev. B: Condens. Matter Mater. Phys., 1996, 54, 77057708.

61 W. Hebenstreit, J. Redinger, Z. Horozova, M. Schmid, R. Podloucky and P. Varga, Surf. Sci., 1999, 424, L321-L328. 62 X. Sun, M. P. Felicissimo, P. Rudolf and F. Silly, Nanotechnology, 2008, 19, 495307.

63 W. Hebenstreit, M. Schmid, J. Redinger, R. Podloucky and P. Varga, Phys. Rev. Lett., 2000, 85, 5376-5379. 